\section{Mudanças no consumo alimentar de mulheres do Município do Rio de Janeiro, Brasil, 1995-2005}

\author{
Changing in dietary intake by women in the \\ Municipality of Rio de Janeiro, Brazil, from \\ 1995 to 2005
}

Rosângela Alves Pereira 1 Roseli Gomes de Andrade 2 Rosely Sichieri 2

\footnotetext{
${ }_{1}^{1}$ Instituto de Nutrição Josué de Castro, Universidade Federal do Rio de Janeiro, Rio de Janeiro, Brasil. 2 Instituto de Medicina Social, Universidade do Estado do Rio de Janeiro, Rio de Janeiro, Brasil.

Correspondência R. A. Pereira

Instituto de Nutrição Josué de Castro, Universidade Federal do Rio de Janeiro. Av. Brigadeiro Trompowski $s / n$, bloco J, $2^{\circ}$ andar, Rio de Janeiro, $R J$ 21941-590, Brasil. rpereira@pobox.com
}

\begin{abstract}
This article compares food intake by women 35 years or older in two population-based cross-sectional studies in Rio de Janeiro, Brazil, in 19951996 ( $n=1,014)$ and 2004-2005 ( $n=1,001)$. Food intake was assessed with a food-frequency questionnaire, and nutritional status was defined according to body mass index (BMI = weight/ height $\left.{ }^{2}\right)$. Prevalence of obesity $\left(B M I \geq 30 \mathrm{~kg} / \mathrm{m}^{2}\right)$ increased in the ten-year period (16.6\% to 24\%). Many high energy density ( $k c a l / g$ ) foods showed a statistically significant increase in the period, such as crackers, candies, bacon, sausage, and hamburger. Some high energy density items were reported less frequently: butter, mayonnaise, potato chips, and sugar. The intake of fruits, milk, beans, roots and potatoes, and meat decreased in the 10-year period. Women with more education showed a larger reduction in fruit and meat intake and a smaller reduction in fish, dairy product, and root and potato intake. Changes in prevalence of obesity were associated with numerous changes in food intake, depending on the level of schooling.
\end{abstract}

Food Consumption; Obesity; Women; Questionnaire

\section{Introdução}

Transição nutricional é a expressão utilizada para sintetizar as mudanças ocorridas no perfil nutricional da população, como a tendência de incremento das prevalências de sobrepeso e obesidade, e que são acompanhadas de modificações na dieta, no padrão de atividade física e nas condições nutricionais 1 . Essas mudanças na alimentação, associadas à redução da atividade física, constituem um dos principais fatores predisponentes para o sobrepeso e obesidade 2,3,4,5.

No Brasil, os dados que permitem avaliar as modificações no consumo de alimentos são as Pesquisas de Orçamentos Familiares (POF), realizadas regularmente pelo Instituto Brasileiro de Geografia e Estatística (IBGE) desde a década de 1970, e que estimam a disponibilidade de alimentos nos domicílios pela avaliação dos gastos com aquisição de alimentos, entretanto, estes inquéritos não permitem a obtenção de informações sobre consumo alimentar individual.

Levy-Costa et al. ${ }^{4}$ observaram que a evolução do padrão alimentar da população brasileira a partir da década de 1970 é consistente com a participação crescente das doenças crônicas nãotrasmissíveis no perfil de morbi-mortalidade da população brasileira e, particularmente, com o aumento da prevalência do excesso de peso e da obesidade no país. Ao avaliar a tendência, comparando as POF realizadas em meados das décadas de 1970, 1980, 1990 e 2000, os autores 
observaram o declínio da aquisição de alimentos tradicionais, como o feijão, o arroz, frutas e hortaliças, e aumento de até $400 \%$ no consumo de produtos industrializados, como biscoitos e refrigerantes. Também foi observada a persistência do consumo excessivo de açúcar e aumento sistemático no teor de gorduras em geral e de gorduras saturadas 4 .

Os potenciais danos para a saúde relacionados a essas alterações no consumo relacionamse com a concentração de energia e com a deficiência e desequilíbrio no teor de nutrientes. Essa configuração da dieta tem sido denominada de "ocidental" ou "ocidentalizada" e engloba, também, o consumo elevado de sal, açúcar e bebidas alcoólicas; redução do consumo de frutas, fibras, verduras e legumes e aumento sistemático no teor de gorduras em geral e de gorduras saturadas. A dieta ocidental vem sendo associada ao incremento da prevalência de excesso de peso e de várias doenças crônicas não-transmissíveis 2,6,7.

Este estudo teve como objetivo avaliar a evolução do consumo alimentar de mulheres com 35 anos ou mais de idade, investigadas em dois estudos de base populacional desenvolvidos no Município do Rio de Janeiro, Brasil, em 19951996 e 2004-2005.

\section{Materiais e métodos}

Para a análise da evolução do consumo alimentar foram comparados dados de dois estudos seccionais, de base populacional, realizados em 1995-1996 e em 2004-2005, que coletaram dados de consumo alimentar, permitindo a análise de tendência de consumo alimentar individual. A população do estudo foi constituída por mulheres, com 35 anos ou mais de idade, residentes no Município do Rio de Janeiro. Foram incluídas mulheres na faixa etária de interesse, residentes nos domicílios particulares permanentes do Município do Rio de Janeiro, não-grávidas ou lactantes.

A pesquisa Nutrição e Saúde no Rio de Janeiro (PNS/RJ), desenvolvida em 1995-1996, investigou amostra probabilística por conglomerados, com dois estágios de seleção; no primeiro, foram selecionados os setores censitários, definidos de acordo com os dados do IBGE para o Município do Rio de Janeiro. No segundo, foram selecionados sistematicamente os domicílios em cada setor. Dos 2.040 domicílios elegíveis, 1.668 participaram da pesquisa. A taxa de não-resposta foi de $18,2 \%$. Foram entrevistados todos os moradores dos domicílios selecionados com idades acima de 12 anos totalizando 4.560 entrevistados, entre os quais 1.401 mulheres na faixa etária de interes- se. Para conferir compatibilidade com o desenho da amostra do estudo de 2004-2005, mantevese na análise apenas uma mulher por domicílio na faixa etária considerada, escolhida aleatoriamente ( $\mathrm{n}=1.075)$ (Figura 1).

Em 2004-2005, a pesquisa Prevalência de Sobrepeso e Consumo Alimentar em Mulheres com 35 Anos ou Mais do Município do Rio de Janeiro considerou como domicílios elegíveis aqueles com pelo menos uma mulher na faixa etária considerada, dentre os domicílios particulares permanentes existentes nos setores censitários determinados no Censo Demográfico de 2000 (http://www.ibge.gov.br) no Município do Rio de Janeiro. O desenho de amostra seguiu o modelo de amostragem probabilística em conglomerados com três estágios de seleção: setor censitário, domicílio e uma mulher em cada domicílio, daquelas acima de 35 anos de idade. $O$ tamanho estimado da amostra foi de 1.200 mulheres. Foram entrevistadas 1.029, o que significou $14 \%$ de perdas (Figura 1).

A pesquisa realizada em 1995-1996 foi desenvolvida respeitando os princípios éticos preconizados na Declaração de Helsinki. A pesquisa de 2004-2005 foi aprovada pelo Comitê de Ética em Pesquisa da Universidade do Estado do Rio de Janeiro em março de 2004

\section{Coleta de dados}

As duas pesquisas obtiveram os dados em entrevistas domiciliares sobre o consumo alimentar, histórico familiar e pessoal de doenças pregressas, dados sócio-econômicos, além da tomada de medidas do peso e da estatura.

Em 1995-1996, o consumo alimentar foi avaliado com a aplicação de um Questionário de Freqüência de Consumo Alimentar (QFCA), com 80 itens, validado para a população adulta do Rio de Janeiro 8, o qual incluía opções abertas para porções. Em 2004-2005, foi utilizado o mesmo questionário com padrão para leitura ótica, porém, modificações no questionário levaram à inclusão de duas ou três opções fechadas de quantidade e a exclusão de dois itens (carne de boi com osso e pão doce). Assim, esta análise contempla a avaliação do consumo dos 78 itens que foram mantidos no QFCA na versão 2004-2005.

Os métodos de coleta dos dados antropométricos (peso e altura) foram similares nas duas pesquisas, com utilização de técnicas padronizadas e pesquisadores treinados. Para a tomada do peso, utilizou-se balança digital marca Filizola (São Paulo, Brasil) em 1995-1996 e Plenna (São Paulo, Brasil) em 2005, em ambas as ocasiões o examinado permanecia com o mínimo de roupa possível e descalço. Em 1995-1996, a estatura foi 
Figura 1

Amostra investigada em dois estudos transversais de base populacional. Município do Rio de Janeiro, Brasil.

1a) $1995-1996$

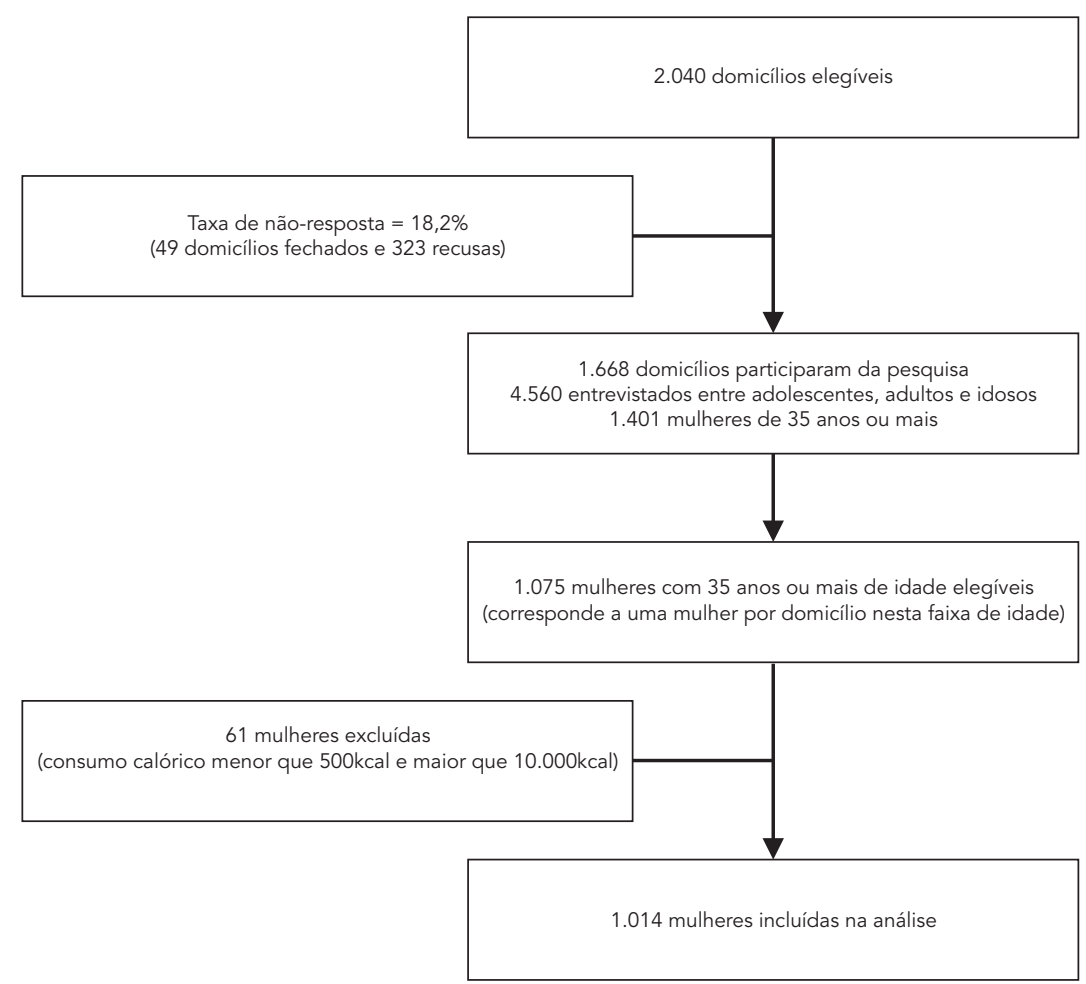

(continua)

aferida com o uso de fita métrica aderida a uma parede sem rodapés e um esquadro de madeira, observando-se as recomendações de Lohman et al. 9 . Em 2005 foi usado estadiômetro marca Seca (Hamburgo, Alemanha) para a tomada da estatura.

Tratamento dos dados de consumo alimentar

A freqüência de consumo relatada para cada item foi transformada em freqüência diária. Para tanto, considerou-se a opção de freqüência "uma vez ao dia" como valor um (1) e procedeu-se à transformação das demais opções em freqüências diárias de forma proporcional (por exemplo, uma vez por semana $=1 / 7$ ). Para estimar a quantidade consumida diariamente (porção diária) de cada alimento, a freqüência diária estimada foi multiplicada pela quantidade consumida (porção referida do alimento).
Para permitir a comparação nos dois momentos foi necessário estabelecer um paralelo (adaptação) entre as porções relatadas em 19951996 de forma aberta, para as duas ou três porções padronizadas que eram as opções para o relato do consumo que constavam na versão do questionário de 2005. Por exemplo, para o arroz na versão de 2004-2005 estavam designadas três opções de porção: duas, três ou quatro colheres de sopa. Para as informantes de 1995-1996, o relato das porções consumidas (pergunta aberta) foi redefinido da seguinte forma: considerou-se como tendo consumido duas colheres de sopa quando era relatado o consumo de até duas colheres de sopa; quando o consumo relatado situava-se entre duas e três colheres, considerou-se a segunda opção (três colheres de sopa) e para relatos de consumo de quatro ou mais colheres de sopa, considerou-se a opção quatro colheres de sopa. 


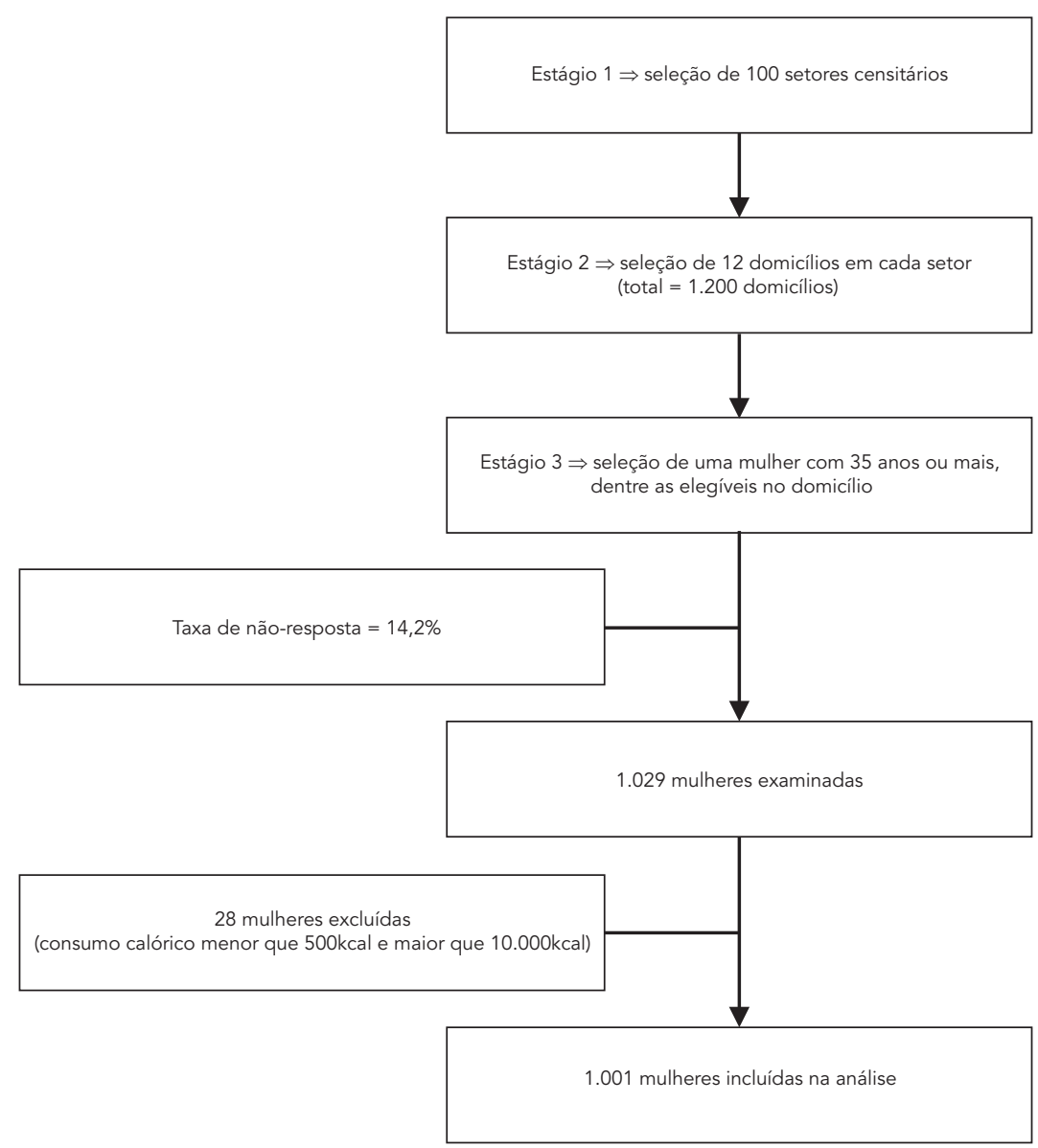

Quanto aos dados de consumo alimentar, foi analisado o consumo de alimentos, grupos de alimentos e alimentos de alta densidade energética. Esses últimos são os que fornecem mais de $1,5 \mathrm{kcal}$ por grama de peso e têm baixo valor nutricional, conforme proposição de Rolls et al. 10 (Tabela 1). Avaliou-se a tendência de consumo considerando a freqüência de consumo diário e semanal (consumo diário x 7) e o número de porções diárias referidas, variáveis analisadas como contínuas. Determinou-se a prevalência do consumo estimando a proporção de indivíduos que referiu consumir cada alimento ou grupo de alimento. Os alimentos consumidos pelo menos duas vezes por semana foram considerados de "consumo usual".

\section{Análise de dados}

A avaliação do estado nutricional nos dois estudos foi realizada com o uso do índice de massa corporal $(\mathrm{IMC}=$ peso/altura 2 ) segundo as recomendações da Organização Mundial da Saúde (OMS) 11, sendo considerado normal para $18,5 \mathrm{~kg} /$ $\mathrm{m}^{2} \leq \mathrm{IMC}<25 \mathrm{~kg} / \mathrm{m}^{2}$, sobrepeso para $25 \mathrm{~kg} / \mathrm{m}^{2} \leq$ IMC $<30 \mathrm{~kg} / \mathrm{m}^{2}$, e obesidade para IMC $\geq 30 \mathrm{~kg} / \mathrm{m}^{2}$.

Para os dois momentos, as mulheres foram estratificadas segundo a faixa de idade, escolaridade (até 4 anos, 5-12 e 13 ou mais anos de estudo) e a renda familiar per capita (considerando-se os múltiplos do salário mínimo segundo os valores praticados em cada um dos períodos em que foi desenvolvida a pesquisa). Realizouse o teste do qui-quadrado para avaliar se havia 
Grupos de alimentos e alimentos de alta densidade energética.

\begin{tabular}{|c|c|}
\hline Grupo & Alimentos incluídos \\
\hline 1 & Leite \\
\hline 2 & Derivados do leite (iogurte, queijo e requeijão) \\
\hline 3 & Arroz \\
\hline 4 & Pão (francês ou de forma) \\
\hline 5 & Produtos à base de cereais (bolo, biscoito doce e biscoito salgado) \\
\hline 6 & Macarrão \\
\hline 7 & Angu e milho \\
\hline 8 & Raízes e tubérculos (aipim, inhame, batata, farinha de mandioca) \\
\hline 9 & Feijão \\
\hline 10 & Frutas (laranja, banana, mamão, maçã, melão, pêra, abacate, abacaxi, goiaba, uva, manga, limão e maracujá) \\
\hline 11 & $\begin{array}{l}\text { Vegetais (alface, couve, repolho, tomate, chicória, quiabo, chuchu, pepino, abóbora, abobrinha, cenoura, vagem, } \\
\text { beterraba, couve-flor e ervilha) }\end{array}$ \\
\hline 12 & Carnes (frango, porco e vaca) \\
\hline 13 & Lingüiça (ou salsicha) \\
\hline 14 & Peixes (peixe, peixe enlatado e camarão) \\
\hline 15 & Açúcar de adição \\
\hline 16 & Doces (sorvete, chocolates, pudim) \\
\hline 17 & Bebidas alcoólicas (vinho, cerveja e outras bebidas alcoólicas - destiladas) \\
\hline 18 & Bebidas adicionadas de açúcar (refrigerante, mate e suco) \\
\hline 19 & Lanches rápidos (pizza, hambúrguer, salgados, chips/batata frita) \\
\hline 20 & Gorduras (manteiga/margarina, bacon) \\
\hline $\begin{array}{l}\text { Alimentos de alta } \\
\text { densidade energética }\end{array}$ & $\begin{array}{l}\text { Maionese, sorvete, bolo, batata frita ou chips, biscoito doce, achocolatado, açúcar, manteiga/margarina, salgadinhos, } \\
\text { chocolate, pizza, pipoca, peixe enlatado, lingüiça/salsicha, biscoito salgado, pudim ou doce, bacon, hambúrguer, balas }\end{array}$ \\
\hline
\end{tabular}

homogeneidade na categorização das mulheres analisadas em 1995-1996 e 2004-2005 de acordo com as variáveis: estado nutricional, idade, grau de escolaridade e renda.

Em pesquisas que utilizam desenhos amostrais complexos, por exemplo, em conglomerados, a variância das estimativas é sempre maior quando é utilizada a amostragem aleatória simples. O cálculo dos estimadores requer programas computacionais específicos que considerem o efeito do desenho amostral. Para as análises estatísticas realizadas, utilizou-se o procedimento survey do programa SAS (SAS Inc., Cary, Estados Unidos) na expansão das estimativas para dados populacionais.

Para descrição das tendências de consumo alimentar foram estimadas as médias (intervalo de 95\% de confiança - IC95\%) das freqüências de consumo e das porções para alimentos e grupos de alimentos nos dois momentos, inclusive considerando os estratos por escolaridade. Para avaliar as possíveis modificações ocorridas no consumo alimentar das mulheres entre as duas pesquisas, por exemplo, se havia diferenças no número de porções de grupos de alimentos consumidos, foram desenvolvidas análises de regressão linear, nas quais foram consideradas como variáveis dependentes o número de porções consumidas diariamente e como variável explanatória, o ano da pesquisa. Os modelos foram ajustados por idade, uma vez que esta foi a única variável associada significativamente com o ano da pesquisa. Essas análises foram realizadas aplicando-se os procedimentos proc surveyreg e proc surveylogit do SAS.

\section{Resultados}

As características das mulheres das duas amostras populacionais modificaram-se no período de dez anos quanto ao estado nutricional, escolaridade e renda. As prevalências de peso normal e sobrepeso reduziram, e a prevalência de obesi- 
Estado nutricional e características das mulheres em inquéritos realizados em 1995-1996 e em 2004-2005. Município do Rio de Janeiro, Brasil.

\begin{tabular}{|c|c|c|c|c|}
\hline & \multicolumn{2}{|c|}{$1995-1996(n=1.014)$} & \multicolumn{2}{|c|}{$2004-2005(n=1.001)$} \\
\hline & $\mathrm{n}$ & $\%$ & $\mathrm{n}$ & $\%$ \\
\hline \multicolumn{5}{|c|}{ Estado nutricional * } \\
\hline Peso normal & 482 & 48,4 & 404 & 43,9 \\
\hline Sobrepeso & 358 & 35,0 & 341 & 32,1 \\
\hline Obesidade & 169 & 16,6 & 254 & 24,0 \\
\hline \multicolumn{5}{|l|}{ Idade (anos) } \\
\hline $35-50$ & 527 & 51,5 & 466 & 45,9 \\
\hline $51-65$ & 309 & 30,2 & 369 & 36,1 \\
\hline$>65$ & 178 & 18,3 & 164 & 18,0 \\
\hline \multicolumn{5}{|c|}{ Escolaridade (anos) * } \\
\hline$\leq 4$ & 664 & 64,0 & 640 & 58,9 \\
\hline $5-12$ & 210 & 21,9 & 224 & 23,4 \\
\hline$\geq 13$ & 128 & 14,2 & 135 & 17,7 \\
\hline \multicolumn{5}{|c|}{ Renda familiar per capita (em salários mínimos) * } \\
\hline Até 2 & 477 & 47,9 & 758 & 72,0 \\
\hline $2-5$ & 295 & 30,6 & 166 & 18,6 \\
\hline 5 ou mais & 178 & 21,4 & 57 & 9,5 \\
\hline
\end{tabular}

* Significância estatística do teste de homogeneidade entre os dois períodos $<0,01$.

dade aumentou de $16,6 \%$ para $24 \%$. Observou-se redução da proporção de mulheres com menos de quatro anos de estudo e aumento daquelas que freqüentaram escola por pelo menos 12 anos. As análises revelaram redução significativa da proporção de mulheres com renda familiar per capita acima de cinco salários mínimos (Tabela 2).

O consumo alimentar avaliado pela prevalência de consumo (referido por pelo menos $70 \%$ das mulheres entrevistadas em 1995-1996), freqüência semanal de consumo e número de porções diárias consumidas pode ser observado nas Tabelas 3, 4 e 5 .

A mudança mais significativa entre os alimentos consumidos com maior freqüência foi observada para o consumo de laranja, que foi referido por $87,1 \%$ das mulheres investigadas em $1995-1996$ e por $66 \%$ das mulheres em 2004-2005, o que representou redução de $24 \%$ na prevalência do consumo $(p<0,001)$. Outros alimentos sofreram variações na freqüência do consumo: cenoura, manteiga/margarina, leite, repolho, suco, açúcar e mamão também foram referidos com menor freqüência na segunda pesquisa. Por outro lado, alimentos como pão, queijo e alface passaram a ser consumidos por maior proporção de mulheres em 2004-2005 (Tabela 3).
Nos dois períodos, os alimentos consumidos pelo menos uma vez por dia (freqüência semanal media $\geq 7,0$ ) eram o açúcar, café, arroz, feijão, manteiga/margarina, pão e leite porém o feijão passou a ser o sétimo alimento mais consumido semanalmente em 2004-2005. Para biscoito salgado, café e tomate observouse elevação significativa da freqüência média de consumo semanal entre 1995-1996 e 2004-2005. Para o arroz, feijão, carne, laranja macarrão, suco, ovo, peixe e queijo foi detectada a redução da freqüência de consumo. Para outros produtos como frango, cenoura, alface, banana, leite, refrigerantes e chuchu, a frequência do consumo manteve-se similar nos dois períodos (Tabela 4 ).

Em 2004-2005, observou-se redução significativa no número médio de porções consumidas diariamente para 11 dos 24 alimentos mais referidos em 1995-1996: feijão, frango, carne bovina laranja, macarrão, suco, leite, ovo, peixe, queijo e refrigerantes. Todavia, o número médio de porções de biscoito salgado consumido diariamente sofreu incremento importante, passando de 0,8 (IC95\%: 0,7-1,0) para 2,1 (IC95\%: 1,9-2,3) (Tabela 5).

A Tabela 6 apresenta as mudanças ocorridas no número de porções diárias consumidas de 
Prevalência de consumo e variação percentual dos alimentos mais referidos por mulheres de 35 anos e mais de idade: comparação entre inquéritos realizados em 1995-1996 e 2004-2005. Município do Rio de Janeiro, Brasil.

\begin{tabular}{|c|c|c|c|c|c|}
\hline \multirow[t]{3}{*}{ Alimentos } & \multicolumn{4}{|c|}{ Prevalência de consumo } & \multirow[t]{3}{*}{ Variação (\%) } \\
\hline & \multicolumn{2}{|c|}{$1995-96$} & \multicolumn{2}{|c|}{ 2004-2005 } & \\
\hline & $\mathrm{n}$ & $\%$ & $\mathrm{n}$ & $\%$ & \\
\hline Arroz * & 989 & 97,5 & 993 & 99,2 & 1,7 \\
\hline Feijão & 961 & 94,8 & 964 & 96,3 & \\
\hline Frango ** & 957 & 94,4 & 966 & 96,5 & 2,2 \\
\hline Café & 908 & 89,5 & 919 & 91,8 & \\
\hline Cenoura ** & 908 & 89,5 & 858 & 85,7 & $-4,2$ \\
\hline Manteiga/Margarina ** & 896 & 88,4 & 844 & 84,3 & $-4,6$ \\
\hline Carne bovina & 891 & 87,9 & 842 & 84,1 & \\
\hline Laranja * & 883 & 87,1 & 661 & 66,0 & $-24,2$ \\
\hline Macarrão & 883 & 87,1 & 886 & 88,5 & \\
\hline Tomate & 881 & 86,9 & 880 & 87,9 & \\
\hline Pão * & 864 & 85,2 & 924 & 92,3 & 8,3 \\
\hline Suco * & 820 & 80,9 & 738 & 73,7 & $-8,9$ \\
\hline Alface ** & 816 & 80,5 & 862 & 86,1 & 7,0 \\
\hline Banana & 805 & 79,4 & 782 & 78,1 & \\
\hline Açúcar * & 803 & 79,2 & 699 & 69,8 & $-11,9$ \\
\hline Leite ** & 803 & 79,2 & 753 & 75,2 & $-5,1$ \\
\hline Ovo & 799 & 78,8 & 757 & 75,6 & \\
\hline Peixe & 796 & 78,5 & 763 & 76,2 & \\
\hline Queijo ** & 761 & 75,0 & 793 & 79,2 & 5,6 \\
\hline Refrigerante & 747 & 73,7 & 710 & 70,9 & \\
\hline Biscoito salgado & 742 & 73,2 & 735 & 73,4 & \\
\hline Chuchu & 733 & 72,3 & 698 & 69,7 & \\
\hline Mamão * & 732 & 72,2 & 597 & 59,6 & $-17,5$ \\
\hline Repolho * & 711 & 70,1 & 646 & 64,5 & $-8,0$ \\
\hline
\end{tabular}

* Teste $\chi^{2} ; p<0,01$;

** Teste $\chi^{2} ; \mathrm{p}<0,05$.

grupos de alimentos. Os resultados indicam que o número de porções de frutas consumido diariamente sofreu redução próxima de $50 \%$, passando de 4,8 em 1995-1996, para 2,6 porções diárias em 2004-2005 ( $\mathrm{p}<0,01$ ). Em 1995-1996, as mulheres consumiam, em média, 2,3 porções diárias de bebidas com adição de açúcar, valor que reduziu para 1,3 em 2004-2005 ( $p<0,01$ ). O consumo dos produtos à base de cereais também sofreu aumento de cerca de $40 \%$ no número de porções consumidas diariamente entre os dois períodos, passando de 1,8 para 2,5 ( $\mathrm{p}<0,01)$. Outros grupos de alimentos experimentaram redução significativa no consumo diário das porções: leite e derivados, macarrão, feijão, carnes, peixes, açúcar, e raízes e tubérculos, enquanto que o número de porções diárias de pão, lingüiça/salsicha e doces aumentou (Tabela 6).

Em função do possível papel dos alimentos de alta densidade energética nas modificações do estado nutricional, avaliou-se a prevalência do consumo usual destes produtos (Tabela 7). Observou-se aumento da prevalência de consumo usual de balas (+351\%), hambúrguer $(+329 \%)$, bacon $(+150 \%)$, pudim ou doce $(+94 \%)$, biscoito salgado $(+72 \%)$, lingüiça/salsicha $(+56 \%)$ e peixe enlatado (+29\%). Houve redução significativa no consumo usual de manteiga/margarina (-6\%), açúcar (-13\%), achocolatado (-19\%), biscoito doce $(-26 \%)$ e batata frita ou chips $(-44 \%)$. 
Freqüência semanal de consumo (médias e intervalos de 95\% de confiança - IC95\%) dos alimentos mais referidos por mulheres de 35 anos e mais de idade em inquéritos realizados em 1995-1996 e em 2004-2005.

Município do Rio de Janeiro, Brasil.

\begin{tabular}{|c|c|c|c|c|}
\hline \multirow[t]{3}{*}{ Alimentos } & \multicolumn{4}{|c|}{ Freqüência semanal } \\
\hline & \multicolumn{2}{|c|}{ 1995-1996 } & \multicolumn{2}{|c|}{ 2004-2005 } \\
\hline & Média & IC95\% & Média & $1 \mathrm{C} 95 \%$ \\
\hline Açúcar & 12,2 & $11,3-13,0$ & 13,2 & $12,4-13,9$ \\
\hline Café & 11,1 & $10,5-11,7$ & 13,1 & $12,7-13,5$ \\
\hline Arroz & 10,6 & $10,1-11,2$ & 9,4 & $9,0-9,8$ \\
\hline Feijão & 9,1 & $8,4-9,8$ & 7,6 & $7,1-8,1$ \\
\hline Manteiga/Margarina & 9,0 & $8,5-9,5$ & 8,6 & $8,2-9,1$ \\
\hline Pão & 8,3 & $7,9-8,8$ & 8,8 & $8,4-9,2$ \\
\hline Leite & 8,2 & $7,8-8,6$ & 8,3 & $7,8-8,8$ \\
\hline Suco & 6,7 & $6,1-7,1$ & 4,8 & $4,4-5,1$ \\
\hline Queijo & 5,7 & $5,2-6,2$ & 4,5 & $4,0-5,0$ \\
\hline Laranja & 5,5 & $5,1-6,0$ & 3,7 & $3,4-4,1$ \\
\hline Banana & 4,8 & $4,4-5,3$ & 4,3 & $3,9-4,7$ \\
\hline Tomate & 4,7 & $4,3-5,1$ & 5,4 & $5,0-5,8$ \\
\hline Refrigerante & 4,4 & $4,0-4,8$ & 4,1 & $3,7-4,6$ \\
\hline Mamão & 4,1 & $3,6-4,5$ & 2,8 & $2,5-3,1$ \\
\hline Carne bovina & 3,9 & $3,5-4,2$ & 2,5 & $2,3-2,6$ \\
\hline Alface & 3,8 & $3,4-4,1$ & 3,9 & $3,6-4,1$ \\
\hline Frango & 3,6 & $3,4-3,9$ & 3,3 & $3,2-3,5$ \\
\hline Cenoura & 2,9 & $2,6-3,2$ & 3,0 & $2,8-3,3$ \\
\hline Chuchu & 2,6 & $2,2-3,0$ & 2,4 & $2,2-2,7$ \\
\hline Macarrão & 2,6 & $2,2-2,9$ & 1,6 & $1,5-1,8$ \\
\hline Biscoito salgado & 2,5 & $2,2-2,8$ & 3,7 & $3,4-4,0$ \\
\hline Ovo & 2,5 & $2,2-2,7$ & 2,0 & $1,8-2,1$ \\
\hline Peixe & 2,0 & $1,6-2,2$ & 1,3 & $1,1-1,4$ \\
\hline Repolho & 2,0 & $1,8-2,3$ & 1,5 & $1,3-1,8$ \\
\hline
\end{tabular}

Verificou-se também aumento estatisticamente significativo da média do número de porções diárias referidas para chocolate/bombom, balas, pudim e biscoito salgado ( $\mathrm{p}<0,05)$, e redução para batata frita e achocolatado $(\mathrm{p}<0,05)$ (dados não apresentados).

Observou-se incremento da ingestão de bebidas alcoólicas entre 1995-1996 e 2004-2005 entre as mulheres com até 12 anos de escolarização; enquanto que as mulheres com 13 ou mais anos de escolarização reduziram o consumo de bebidas alcoólicas no período considerado, embora nenhuma destas alterações tenha sido estatisticamente significativa. O consumo de frutas mostrou alterações significativas $(p<0,05)$ segundo o grau de escolaridade: aumentou no grupo de até 4 anos de escolarização e reduziu de maneira importante nos grupos com 5-12 anos e 13 ou mais anos de escolarização. O consumo de bebidas adicionadas de açúcar (que inclui os refrigerantes) reduziu nos três estratos de grau de escolaridade ( $\mathrm{p}<0,05)$ (dados não mostrados).

Observaram-se singularidades na variação do consumo pelas mulheres investigadas quando se considerou o grau de escolaridade. Aquelas de menor escolaridade ( $\leq 4$ anos de estudo) apresentaram aumento $(\mathrm{p}<0,05)$ no consumo de frutas e lingüiça/salsicha, e redução de leite e derivados, carnes e feijão. As mulheres que tinham entre 5 e 12 anos de estudo apresentaram aumento $(\mathrm{p}<0,05)$ no consumo de bolo e biscoitos, pão e gorduras (manteiga/margarina e bacon) e 
Número de porções diárias (médias e intervalos de 95\% de confiança - IC95\%) dos alimentos mais referidos por mulheres de 35 anos e mais de idade em inquéritos realizados em 1995-1996 e em 2004-2005. Município do Rio de Janeiro, Brasil.

\begin{tabular}{|c|c|c|c|c|}
\hline \multirow[t]{3}{*}{ Alimentos } & \multicolumn{4}{|c|}{ Número de porções diárias } \\
\hline & \multicolumn{2}{|c|}{$1995-1996$} & \multicolumn{2}{|c|}{ 2004-2005 } \\
\hline & Média & IC95\% & Média & IC95\% \\
\hline Arroz & 4,5 & $4,2-4,9$ & 4,3 & $4,1-4,6$ \\
\hline Açúcar & 3,6 & $3,2-3,9$ & 3,1 & $2,8-3,4$ \\
\hline Café & 2,3 & $2,1-2,5$ & 2,1 & $2,0-2,2$ \\
\hline Alface & 1,8 & $1,6-2,0$ & 2,0 & $1,9-2,2$ \\
\hline Feijão & 1,8 & $1,6-2,0$ & 1,3 & $1,2-1,4$ \\
\hline Pão & 1,6 & $1,5-1,7$ & 1,6 & $1,5-1,7$ \\
\hline Laranja & 1,5 & $1,3-1,6$ & 0,8 & $0,7-0,9$ \\
\hline Leite & 1,5 & $1,4-1,6$ & 1,3 & $1,2-1,4$ \\
\hline Queijo & 1,4 & $1,2-1,5$ & 1,0 & $0,9-1,2$ \\
\hline Manteiga/Margarina & 1,3 & $1,2-1,4$ & 1,2 & $1,2-1,3$ \\
\hline Suco & 1,3 & $1,2-1,5$ & 0,8 & $0,7-0,9$ \\
\hline Banana & 1,1 & $1,0-1,2$ & 0,8 & $0,7-0,9$ \\
\hline Refrigerante & 1,0 & $0,9-1,2$ & 0,8 & $0,7-0,9$ \\
\hline Tomate & 1,0 & $0,8-1,1$ & 0,9 & $0,8-0,9$ \\
\hline Cenoura & 0,9 & $0,8-1,0$ & 1,0 & $0,9-1,1$ \\
\hline Frango & 0,9 & $0,8-0,9$ & 0,7 & $0,7-0,7$ \\
\hline Biscoito salgado & 0,8 & $0,7-1,0$ & 2,1 & $1,9-2,3$ \\
\hline Carne bovina & 0,7 & $0,6-0,8$ & 0,4 & $0,4-0,4$ \\
\hline Chuchu & 0,7 & $0,6-0,8$ & 0,8 & $0,7-0,9$ \\
\hline Macarrão & 0,7 & $0,6-0,8$ & 0,5 & $0,5-0,6$ \\
\hline Mamão & 0,7 & $0,6-0,8$ & 0,5 & $0,4-0,6$ \\
\hline Repolho & 0,6 & $0,5-0,7$ & 0,5 & $0,4-0,6$ \\
\hline Ovo & 0,5 & $0,5-0,6$ & 0,4 & $0,3-0,4$ \\
\hline Peixe & 0,4 & $0,3-0,5$ & 0,3 & $0,2-0,3$ \\
\hline
\end{tabular}

redução no consumo de peixes, cereais, feijão, carnes, leite e derivados, e frutas. As mulheres com 13 e mais anos de estudo aumentaram $(\mathrm{p}<$ 0,05 ) o consumo de doces (sorvete, pudim e chocolate em barra e em pó), pão, cereais, lanches rápidos (pizza, hambúrguer, salgadinhos e batata frita) e lingüiça/salsicha, e reduziram ( $\mathrm{p}<$ 0,05 ) o consumo de leite, carnes e frutas (dados não mostrados).

\section{Discussão}

A elevação da prevalência do excesso de peso no período entre 1995-1996 e 2004-2005 em mulheres do Rio de Janeiro é consistente com os resultados de outros estudos realizados no Brasil 12,13. Em estudo anterior foram discutidas as variações no consumo de macronutrientes e a evolução das taxas de obesidade nessa população, observando-se o incremento no consumo de gorduras no estrato de escolaridade mais elevada e a sua redução entre as mulheres com baixo nível de escolarização 14 . Esses achados motivaram a investigação mais detalhada do consumo de alimentos e de grupos de alimentos, tendo em vista a compreensão das variações na ingestão de macronutrientes.

As variações no consumo de alimentos de mulheres com idades acima de 35 anos do Rio de Janeiro entre 1995-1996 e 2004-2005 caracterizaram-se pela redução da qualidade nutricional da 
Médias do número de porções diárias de grupos de alimentos e coeficientes de regressão linear ajustados por idade ( $\beta$ ) de mulheres de 35 anos e mais de idade em inquéritos realizados em 1995-1996 e em 2004-2005. Município do Rio de Janeiro, Brasil.

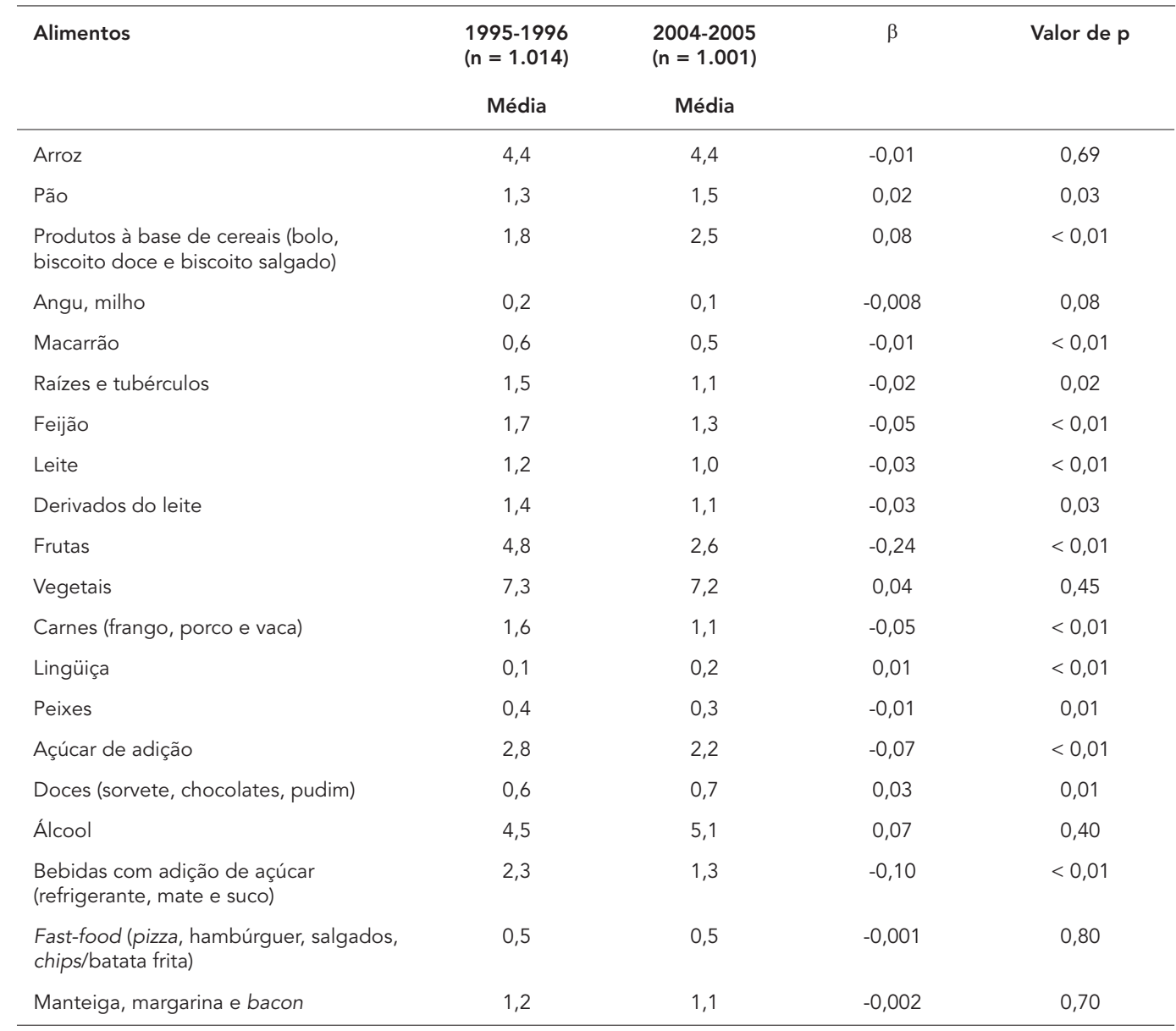

dieta. Observou-se notável redução no consumo de alimentos de alto valor nutricional como frutas, feijão e laticínios, e o incremento no consumo de itens como o biscoito salgado. Porém, é interessante apontar, entretanto, a diminuição do consumo de alimentos como a batata frita, os refrigerantes e o açúcar.

O consumo geral de frutas reduziu, entretanto, na análise estratificada por escolaridade, observou-se aumento do consumo de frutas entre as mulheres de baixo grau de instrução e a redução no consumo deste grupo de alimentos entre aquelas com 5 ou mais anos de escolarização. Uma hipótese seria atribuir esses resultados à ocorrência de um possível erro de classificação, dado que o relato do número de porções consumidas no inquérito de 1995-1996 era feito com base em uma questão aberta, e em 2005 com base em três opções fechadas. Contudo, outros itens deveriam sofrer o mesmo problema; isso não ocorreu, por exemplo, com o relato do consumo de vegetais, sugerindo que houve modificação real no consumo de frutas entre mulheres nessa faixa de idade.

O número de porções de frutas consumidas pelas mulheres de baixa escolaridade em 19951996 era menos da metade daquele consumido pelas mulheres com pelo menos 5 anos de estudo. Assim, o modesto incremento no consumo de frutas observado no grupo de baixa escolaridade possivelmente relaciona-se com o aumento da disponibilidade destes alimentos no mercado 15 e a redução relativa do seu custo, aliados ao aumento do poder de compra ocorrido no país a partir de 200316 .

As modificações do consumo alimentar de mulheres com elevado grau de escolarização indicam o aumento do consumo de alimentos poupa- 
Prevalência do consumo usual de alimentos de alta densidade energética referido por mulheres de 35 anos e mais de idade em inquéritos realizados em 1995-1996 e em 2004-2005. Município do Rio de Janeiro, Brasil.

\begin{tabular}{lccc}
\hline Alimentos & \multicolumn{2}{c}{ Prevalência de consumo } & Variação (\%) \\
& $1995-1996$ & $\mathbf{2 0 0 4 - 2 0 0 5}$ & \\
\hline Manteiga/Margarina * & 83,0 & 78,0 & -6 \\
Açúcar * & 76,6 & 67,0 & -13 \\
Biscoito doce * & 32,0 & 23,6 & -26 \\
Biscoito salgado ** & 29,0 & 50,0 & 72 \\
Batata frita ou chips * & 19,0 & 10,7 & -44 \\
Bolo & 13,5 & 7,5 & -59 \\
Maionese * & 12,6 & 5,2 & -19 \\
Achocolatado * & 13,4 & 10,9 & 94 \\
Sorvete & 10,5 & 5,0 & 56 \\
Pudim ou doce ** & 6,7 & 13,0 & 329 \\
Lingüiça/Salsicha * & 9,0 & 14,0 & \\
Hambúrguer ** & 3,5 & 15,0 & 29 \\
Pizza & 2,6 & 2,8 & \\
Chocolate & 6,0 & 6,0 & 351 \\
Salgadinhos & 6,0 & 6,0 & \\
Balas ** & 4,1 & 18,5 & \\
Pipoca & 5,0 & 6,4 & 10,0 \\
Bacon ** & 4,0 & 2,7 & \\
Peixe enlatado * & 2,1 & & \\
\hline
\end{tabular}

* Teste $\chi^{2} ; p<0,05$;

** Teste $\chi^{2} ; p<0,01$.

dores de tempo (lanches, pão, lingüiça/salsicha), que além de alta densidade energética, apresentam baixa densidade de micronutrientes.

A redução no consumo de carnes e peixes e aumento no delingüiça/salsicha também são modificações preocupantes, uma vez que as carnes e peixes são fontes mais saudáveis de proteínas do que as carnes preservadas. Aumento da disponibilidade domiciliar de embutidos no Brasil também foi documentado nos dados de orçamento familiar 4 .

A redução do consumo de alimentos básicos tradicionais, principalmente o feijão, entre as mulheres do Rio de Janeiro, pode ser considerada característica típica do processo de transição nutricional, apropriadamente descrito por Tardido \& Falcão ${ }^{17}$. O efeito desse tipo de mudança sobre o perfil de saúde e nutrição vem sendo relacionado ao aumento da prevalência de obesidade e de distúrbios metabólicos, que são, em última instância, fatores de risco importantes para as doenças crônicas não-transmissíveis, como as cardiovasculares e o câncer. $\mathrm{O}$ abandono progressivo da dieta tradicional brasileira tem sido relatado nos estudos de consumo alimentar 4,18,19. Estudo de corte transversal realizado por Sichieri 20 no Município do Rio de Janeiro, indicou que a dieta tradicional brasileira, baseada em arroz e feijão, era protetora para sobrepeso e obesidade.

A redução no consumo de bebidas com adição de açúcar e de biscoito doce poderia indicar um possível viés de informação na direção de uma alimentação com menor valor energético, dado que já é do senso comum o conhecimento de que estes alimentos são fontes de energia e podem estar relacionados ao ganho de peso. Esses dados poderiam, portanto, indicar que as mulheres estariam restringindo o consumo de alimentos considerados "vilões" na luta contra o ganho de peso, motivado pelo fato de já se encontrarem com excesso de peso ou como medida de prevenção para o excesso de peso.

$\mathrm{O}$ aumento no consumo de biscoitos salgados pode ser indicativo de estratégia, não muito eficiente, de perda de peso, que seria a substituição 
do pão por crackers. Pode também refletir o fato de que as populações de grandes centros urbanos permanecem longos períodos fora de casa e optam freqüentemente por lanches rápidos, de custo relativamente baixo e fáceis de portar, o que vem a ser o caso dos biscoitos salgados.

O incremento no consumo de alimentos de alta densidade energética é coerente com as variações observadas nas taxas de obesidade entre essas mulheres. Resultados semelhantes foram obtidos por Nielsen et al. 21, que analisaram a variação do consumo energético nos Estados Unidos entre 1977 e 1996 e evidenciaram o aumento do consumo de alimentos de alta densidade energética e de baixa qualidade nutricional, como pizza, em todas as faixas etárias acompanhando o crescimento do excesso de peso naquele país.

A avaliação do consumo de alimentos de alta densidade energética é relevante, dado que independente do conteúdo lipídico, estes alimentos aumentam a ingestão calórica total 22 e têm efeito deletério sobre a saúde, como tem sido evidenciado em diversas investigações. Alguns autores têm buscado elucidar o efeito do consumo desses alimentos sobre a regulação do apetite e ingestão calórica 23,24. Estudo de Mendoza et al. 25 encontrou associação entre consumo de alimentos de alta densidade energética com obesidade e síndrome metabólica em amostra de base populacional de adultos nos Estados Unidos. Schroder et al. 26 avaliaram a relação entre consumo de fast-food e obesidade em amostra de base populacional da Espanha e encontraram associação entre freqüência de consumo destes alimentos com o aumento do risco de obesidade. Outros estudos que abordam a relação positiva entre ingestão de alimentos de alta densidade energética e obesidade sugerem que o incentivo do consumo de frutas e vegetais é uma importante estratégia para reverter este quadro 27,28,29,30.

Embora esses resultados sejam de grande importância para o desenvolvimento de ações de promoção da alimentação saudável e prevenção do ganho de peso, este estudo tem como limitação o fato de não apresentar dados de atividade física ou de gasto de energia. Não foi possível identificar um padrão claro que pudesse explicar por que as mulheres de maior escolaridade apresentaram menor incremento na prevalência de obesidade, o que poderia ser explicado, possivelmente, por diferentes padrões de gasto energético entre os estratos por nível de instrução.

Outras limitações que podem ser atribuídas ao estudo, poderiam estar relacionadas com o instrumento utilizado para a investigação do consumo alimentar, o questionário de freqüência do consumo de alimentos. Entretanto, considera-se que, nesta investigação especificamente, esse instrumento mostrou-se de grande valia, pois sendo validado para a população em estudo e adequado para a categorização do consumo em gradientes de intensidade (prevalência do consumo, freqüência de consumo, número de porções) permite a comparação dos dois momentos investigados. Além disso, quaisquer vieses (sub ou super-relatos) estariam sendo observados para os dois momentos do estudo, propiciando a comparação de dados similares.

Este estudo mostrou que as modificações no consumo alimentar por mulheres adultas da Região Metropolitana do Rio de Janeiro se contrapõem às orientações para uma alimentação saudável 31. Tais mudanças afetam a qualidade nutricional da alimentação desse grupo da população, e possivelmente contribuem para a ocorrência do sobrepeso e da obesidade, além de concorrerem para o desenvolvimento de distúrbios metabólicos, como a síndrome metabólica e as dislipidemias, e outras doenças crônicas nãotransmissíveis. Os resultados indicam a necessidade de priorização das ações de promoção da saúde voltadas para essas mulheres, particularmente, considerando que, usualmente, a decisão das mulheres é definitiva na composição da alimentação familiar. 


\section{Resumo}

Compara-se o consumo alimentar de mulheres de 35 anos e mais investigado em dois estudos transversais de base populacional desenvolvidos no Rio de Janeiro, Brasil, em 1995-1996 ( $n=1.014)$ e 2004-2005 ( $n=$ 1.001). Utilizou-se questionário de freqüência do consumo de alimentos para estimar o consumo de alimentos e o indice de massa corporal $(I M C=$ pesolesta tura 2 ) para avaliar o estado nutricional. A prevalência da obesidade (IMC $\geq 30 \mathrm{~kg} / \mathrm{m}^{2}$ ) aumentou no período de 10 anos (16,6\% para 24\%). O consumo de diversos alimentos com alta densidade energética sofreu incremento, como: biscoitos salgados, doces, bacon, lingüiça/salsicha e hambúrguer. Outros foram relatados com menor freqüência: manteiga/margarina, maionese, batata frita ou chips, e açúcar. Frutas, leite, feijão, raízes e tubérculos, e carnes tiveram redução do consumo. Mulheres com escolaridade elevada apresentaram maior redução no consumo de frutas e carnes. As mudanças na prevalência da obesidade parecem estar relacionadas com as modificações do consumo de alimentos e dependentes do grau de escolarização.

Consumo de Alimentos; Obesidade; Mulheres; Questionário

\section{Referências}

1. Popkin BM. The nutrition transition and obesity in the developing world. J Nutr 2001; 131:871S-3S.

2. Monteiro CA, Mondini L, Costa RBL. Mudanças na composição e adequação nutricional da dieta familiar nas áreas metropolitanas do Brasil (19881996). Rev Saúde Pública 2000; 34:251-8.

3. Mendonça CP, Anjos LA. Aspectos das práticas alimentares e da atividade física como determinantes do crescimento do sobrepeso/obesidade no Brasil. Cad Saúde Pública 2004; 20:698-709.

4. Levy-Costa RB, Sichieri R, Pontes NDS, Monteiro CA. Disponibilidade domiciliar de alimentos no Brasil: distribuição e evolução (1974-2003). Rev Saúde Pública 2005; 39:530-40.

5. Astrup A, Dyerberg J, Selleck M, Stender S. Nutrition transition and its relationship to the development of obesity and related chronic diseases. Obes Rev 2008; 9 Suppl 1:48-52.

\section{Colaboradores}

R. A. Pereira participou na concepção do estudo, análise e interpretação dos dados, e redação do manuscrito. R. G. Andrade colaborou na coleta, análise e interpretação de dados, e redação do manuscrito. R. Sichieri contribuiu na concepção e desenho do estudo, análise e interpretação dos dados, e redação do manuscrito.

\section{Agradecimentos}

Ao Conselho Nacional de Desenvolvimento Científico e Tecnológico (CNPq) e à Coordenação de Aperfeiçoamento de Pessoal de Nível Superior (CAPES). À Fundação de Amparo à Pesquisa do Estado do Rio de Janeiro (FAPERJ), Ministério da Saúde, e ao Laboratório Abbott do Brasil pelo financiamento.
6. Filozof C, Gonzalez C, Sereday M, Mazza C, Braguinsky J. Obesity prevalence and trends in LatinAmerican countries. Obes Rev 2001; 2:99-106.

7. French SA, Story M, Jeffery RW. Environmental influences on eating and physical activity. Annu Rev Public Health 2001; 22:309-35.

8. Sichieri R, Everhart JE. Validity of a Brazilian food frequency questionnaire against dietary recalls and estimated energy intake. Nutr Res 1998; 18:1649-59.

9. Lohman TG, Roche AF, Martorell R. Anthropometric standardization reference manual. Champaign: Human Kinetics Books; 1988.

10. Rolls BJ, Bell EA, Castellanos VH, Chow M, Pelkman CL, Thorwart ML. Energy density but not fat content of foods affected energy intake in lean and obese women. Am J Clin Nutr 1999; 69:863-71. 
11. World Health Organization. Obesity: preventing and managing the global epidemic. Geneva: World Health Organization; 1997. (WHO Technical Report Series, 894).

12. Instituto Brasileiro de Geografia e Estatística. Análise da disponibilidade domiciliar de alimentos e do estado nutricional no Brasil: Pesquisa de Orçamentos Familiares 2002-2003. Rio de Janeiro: Instituto Brasileiro de Geografia e Estatística; 2004.

13. Monteiro CA, Conde WL, Popkin BM. Income-specific trends in obesity in Brazil: 1975-2003. Am J Public Health 2007; 97:1808-12.

14. Andrade RG, Pereira RA, Sichieri R. Ten-year increase in the prevalence of obesity and reduction of fat intake in Brazilian women. J Epidemiol Community Health; no prelo.

15. Claro RM, Carmo HCE, Machado FMS, Monteiro CA. Renda, preço dos alimentos e participação de frutas e hortaliças na dieta. Rev Saúde Pública 2007; 41:557-64.

16. Instituto Brasileiro de Análises Sociais e Econômicas. Repercussões do Programa Bolsa Família na segurança alimentar e nutricional das famílias beneficiadas. http://www.ibase.br/userimages/ ibase_bf_sintese_site.pdf (acessado em 27/ Mar/2009)

17. Tardido AP, Falcão MC. O impacto da modernização na transição nutricional e obesidade. Rev Bras Nutr Clín 2006; 21:117-24.

18. Jaime PC, Monteiro CA. Fruit and vegetable intake by Brazilian adults, 2003. Cad Saúde Pública 2005; 21 Suppl 1:S19-24.

19. Lopes ACS, Caiaffa WT, Sichieri R, Mingoti SA, Lima-Costa MF. Consumo de nutrientes em adultos e idosos em estudo de base populacional: Projeto Bambuí. Cad Saúde Pública 2005; 21:1201-9.

20. Sichieri R. Dietary patterns and their associations with obesity in the Brazilian city of Rio de Janeiro. Obes Res 2002; 10:42-8.

21. Nielsen SJ, Siega-Riz AM, Popkin BM. Trends in energy intake in U.S. between 1977 and 1996: similar shifts seen across age groups. Obes Res 2002; 10:370-8.
22. Rolls BJ, Bell EA. Intake of fat and carbohydrate: role of energy density. Eur J Clin Nutr 1999; 53 Suppl 1:S166-73.

23. Prentice AM, Jebb SA. Fast foods, energy density and obesity: a possible mechanistic link. Obes Rev 2003; 4:187-94.

24. McCrory MA, Saltzman E, Rolls BJ, Roberts SB. A twin study of the effects of energy density and palatability on energy intake of individual foods. Physiol Behav 2006; 87:451-9.

25. Mendoza JA, Drewnowski A, Christakis DA. Dietary energy density is associated with obesity and the metabolic syndrome in U.S. adults. Diabetes Care 2007; 30:974-9.

26. Schroder H, Fito M, Covas MI. Association of fast food consumption with energy intake, diet quality, body mass index and the risk of obesity in a representative Mediterranean population. Br J Nutr 2007; 98:1274-80.

27. Rolls BJ, Ello-Martin JA, Tohill BC. What can intervention studies tell us about the relationship between fruit and vegetable consumption and weight management? Nutr Rev 2004; 62:1-17.

28. Rolls BJ, Roe LS, Beach AM, Kris-Etherton PM. Provision of foods differing in energy density affects long-term weight loss. Obes Res 2005; 13:1052-60.

29. Ledikwe JH, Blanck HM, Khan LK, Serdula MK, Seymour JD, Tohill BC, et al. Low-energy-density diets are associated with high diet quality in adults in the United States. J Am Diet Assoc 2006; 106:1172-80.

30. Oliveira MC, Sichieri R, Venturim-Mozzer R. A lowenergy-dense diet adding fruit reduces weight and energy intake in women. Appetite 2008; 51:291-5.

31. Ministério da Saúde. Guia alimentar para a população brasileira: promovendo a alimentação saudável. Brasília: Ministério da Saúde; 2005.

Recebido em 22/Dez/2008

Versão final reapresentada em 06/Jul/2009

Aprovado em 06/Ago/2009 